\title{
Das Fazit: Nutzen und Grenzen der Vertriebs-Balanced Scorecard
}

Eine Vertriebs-Balanced Scorecard hilft, von der Vertriebsstrategie ausgehend die Aktionsfelder des Vertriebs in eine sinnvolle Balance zu bringen. Resultat ist ein Maßnahmenpaket, das per Kennzahlensystem gesteuert wird. Kritikpunkte an der Balanced Scorecard gibt es viele und kaum eine Publikation lässt den erhobenen Zeigefinger aus, mit dem warnend darauf hingewiesen wird, dass eine Balanced Scorecard methodisch sauber und konsequent anzuwenden sei, damit sie funktioniere. Dem ist nichts hinzuzufügen.

In der betrieblichen Praxis scheitern Vertriebs-Balanced Scorecards in der Mehrzahl der Fälle, weil sie methodisch unsauber aufgebaut sind oder der Aufwand der ständigen Pflege gescheut wird. Sie werden als Workshop-Tool genutzt, nicht aber als Instrument der permanenten Vertriebssteuerung. Oftmals erodieren sie zu Kennzahlenlisten und die Zielsetzung, allen Vertriebsmitarbeitern die Arbeit und insbesondere dem Management die Führung durch klare Vorgaben zu erleichtern, wird nicht erfüllt. Dass sie niemals mehr sind, als die unvollkommene Abbildung eines komplexen Geschehens, das nur bedingt der eigenen Kontrolle unterliegt und von einer unberechenbaren Umweltdynamik beeinflusst wird, versteht sich von selbst. Doch ohne solche Methoden gelingt es nicht, ein Unternehmen - oder eine größere Vertriebsorganisation - zu führen. 